PACS 04.25.Nx

\title{
MANY-PARTICLE CORRELATION EFFECTS AND MAGNETISM OF WEAKLY RELATIVISTIC ELECTRON GAS
}

\author{
L.F.Blazhyjevskii, H.B.Hil', Yu.S.Krynytskyi \\ Ivan Franko Lviv State University \\ Department for Theoretical Physics \\ 12 Dragomanov St., UA-290005, Lviv-05, Ukraine
}

Received February 24, 1997

\begin{abstract}
The path integral quantum-statistical approach to study the magnetic properties of the weakly relativistic interacting electron gas in the inhomogeneous magnetic field is suggested. In the random phase approximation the thermodynamic potential is calculated. In the case of absolute zero the magnetization and nonlinear magnetic susceptibility of the system are found. Both Landau diamagnetism and Pauli paramagnetism are taken into account. It is shown that in the region of high electron density the relativistic contributions are essential and lead to the increase of magnetic susceptibility.
\end{abstract}

Classical and quantum treatment of the magnetic properties of relativistic ideal electron gas was given in ref. [1,2]. The many-particle corrections (including correlation contributions) to the magnetic susceptibility of the non-relativistic electron gas are discussed in ref. [3]. For the relativistic description of the interacting charged spin particles the spin-spin, spin-orbital, contact, Coulomb and retarded electromagnetic interactions, as well as the interaction with external field are necessary to take into account. Explicit form of the relativistic interactions is complicated and consequently the construction of the corresponding statistical theory is complicated, as well. However for the weakly relativistic case there is a possibility of statistical description analogous to the non-relativistic theory [4].

In the present paper an approach to the quantum-statistical theory of the magnetism of the interacting weakly relativistic electron gas is suggested. Such approach is based on the Lagrange description and path integral formalism. This formalism was developed in papers $[4,5]$.

The Lagrangian of the weakly relativistic electron gas in the presence of the external inhomogeneous magnetic field $\boldsymbol{H}_{0}(\boldsymbol{r})=\operatorname{rot} \boldsymbol{A}^{0}(\boldsymbol{r})$ is as follows

$$
L=L_{D}+L_{C}+L_{S O}+L_{S S}+L_{F} .
$$

The term

$$
L_{D}=\sum_{i=1}^{N}\left(\frac{m v_{i}^{2}}{2}+\frac{m v_{i}^{4}}{8 c^{2}}\right)-\frac{1}{2} \sum_{i \neq j}^{N} \frac{e^{2}}{r_{i j}}\left\{1-\frac{1}{2 c^{2}}\left[\left(\boldsymbol{v}_{i}, \boldsymbol{v}_{j}\right)+\frac{\left(\boldsymbol{r}_{i j}, \boldsymbol{v}_{i}\right)\left(\boldsymbol{r}_{i j}, \boldsymbol{v}_{j}\right)}{r_{i j}^{2}}\right]\right\}
$$


is Darwin Lagrangian that describes Coulomb and electromagnetic interactions,

$$
L_{C}=\frac{1}{2} \sum_{i \neq j}^{N} \frac{\pi e^{2} \hbar^{2}}{m^{2} c^{2}} \delta\left(\boldsymbol{r}_{i j}\right)
$$

corresponds to the Darwin contact interactions. The contributions

$$
\begin{gathered}
L_{S O}=\frac{1}{2} \sum_{i \neq j}^{N} \frac{e^{2} \hbar}{2 m c^{2} r_{i j}^{3}}\left(\boldsymbol{\sigma}_{i}\left[\boldsymbol{r}_{i j},\left(\boldsymbol{v}_{i}-2 \boldsymbol{v}_{j}\right)\right]\right), \\
L_{S S}=-\frac{1}{2} \sum_{i \neq j}^{N} \frac{e^{2} \hbar^{2}}{4 m^{2} c^{2}}\left\{\frac{\left(\boldsymbol{\sigma}_{i}, \boldsymbol{\sigma}_{j}\right)}{r_{i j}^{3}}-3 \frac{\left(\boldsymbol{\sigma}_{i}, \boldsymbol{r}_{i j}\right)\left(\boldsymbol{\sigma}_{j}, \boldsymbol{r}_{i j}\right)}{r_{i j}^{5}}-\frac{8 \pi}{3}\left(\boldsymbol{\sigma}_{i}, \boldsymbol{\sigma}_{j}\right) \delta\left(\boldsymbol{r}_{i j}\right)\right\}
\end{gathered}
$$

describe spin-orbit and spin-spin interactions, respectively. Interaction with the external field is determined by Lagrangian

$$
L_{F}=\sum_{i=1}^{N} \frac{e}{c}\left(\left(\boldsymbol{v}_{i}, \boldsymbol{A}^{0}\left(\boldsymbol{r}_{i}\right)\right)+\frac{\hbar}{2 m}\left(\boldsymbol{\sigma}_{i}, \boldsymbol{H}_{0}\left(\boldsymbol{r}_{i}\right)\right)\right) .
$$

Here $N$ is a number of electrons, $e$ is the electronic charge, $m$ is the electronic mass, $c$ is the speed of light, $\boldsymbol{r}_{i}$ and $\boldsymbol{v}_{i}$ are the position and velocity of the $i$-th electron, respectively, $\boldsymbol{r}_{i j}=\boldsymbol{r}_{i}-\boldsymbol{r}_{j}$. The quantities $\boldsymbol{\sigma}_{i}$ are Pauli spin matrices for particle $i, \delta(\boldsymbol{r})$ is Dirac $\delta$-function.

The thermodynamic potential $\Omega$ of the quantum charges system (1) at the statistical temperature $\beta^{-1}$ can be represented in the form of path integral [5]:

$$
\begin{aligned}
& \mathrm{e}^{-\beta \Omega}=\operatorname{reg} A \int \mathcal{D} \boldsymbol{R}_{\boldsymbol{k}}(\tau) \exp \left\{\frac{1}{2} \sum_{\boldsymbol{k}} \int_{0}^{\beta} \mathrm{d} \tau\left(\boldsymbol{R}_{\boldsymbol{k}}(\tau), \boldsymbol{R}_{-k}(\tau)\right)\right\} \mathrm{e}^{-\beta \Omega(R)}, \\
& -\beta \Omega(R)=\sum_{n \geqslant 1} \frac{(-1)^{n+1}}{n} \mathrm{e}^{n \beta \mu} Z_{1}(\beta n) .
\end{aligned}
$$

Here $A$ is a normalizing factor, which can be find after equalizing of integral (2) to one under $Z_{1}=0$ condition, $\mu$ is chemical potential. Symbol "reg" denotes that the contributions proportional to $[\delta(0)]^{l}$ are neglected after the calculation of all path integrals $(l=1,2, \ldots)$ was performed. $Z_{1}(\beta n)$ can be treated as partition function of one particle in the same fictitious external field $\boldsymbol{R}_{\boldsymbol{k}}(\tau)$ at temperature $\Theta=1 / n \beta$. This partition function can be determined by the next expressions

$$
\begin{aligned}
& Z_{1}(\beta n)=\operatorname{Sp} \int \mathrm{d}^{3} r K(\boldsymbol{r}, \boldsymbol{r} ; n \beta), \\
& K\left(\boldsymbol{r}, \boldsymbol{r}^{\prime} ; \beta\right)=\operatorname{reg} C \int \mathcal{D}^{3} u(\tau) \mathrm{e}^{\left(S_{0}+S^{\prime}+S_{F}\right)} \delta\left(\boldsymbol{r}-\boldsymbol{r}^{\prime}-\hbar \int_{0}^{\beta} \mathrm{d} \tau \boldsymbol{u}(\tau)\right), \\
& S^{0}=\int_{0}^{\beta} \mathrm{d} \tau\left\{-\frac{m u^{2}(\tau)}{2}-\frac{m u^{4}(\tau)}{8 c^{2}}+\right.
\end{aligned}
$$




$$
\begin{aligned}
& \left.+\frac{1}{2} \sum_{\boldsymbol{k}} \frac{4 \pi e^{2}}{V k^{2}}\left(1+\frac{2 u^{2}(\tau)}{3 c^{2}}-\frac{3 \hbar^{2} k^{2}}{4 m^{2} c^{2}}\right)\right\}, \\
& S^{\prime}=\int_{0}^{\beta} \mathrm{d} \tau \sum_{\boldsymbol{k}} i e\left(\frac{4 \pi}{V k^{2}}\right)^{1 / 2}\left(\boldsymbol{R}_{\boldsymbol{k}}(\tau), \boldsymbol{F}_{\boldsymbol{k}}(\tau)\right) \mathrm{e}^{i \boldsymbol{k} \boldsymbol{r}(\tau)}, \\
& S_{F}=\int_{0}^{\beta} \mathrm{d} \boldsymbol{r} \sum_{\boldsymbol{k}} \frac{i e}{c V}\left(\left(\boldsymbol{A}_{\boldsymbol{k}}^{0}, \boldsymbol{u}(\tau)\right)+\frac{\hbar}{2 m}\left(\left[\boldsymbol{k}, \boldsymbol{A}_{\boldsymbol{k}}^{0}\right] \boldsymbol{\sigma}\right)\right) \mathrm{e}^{i \boldsymbol{k} \boldsymbol{r}(\tau)}, \\
& \boldsymbol{F}_{\boldsymbol{k}}(\tau)=\frac{\boldsymbol{k}}{k}\left(1-\frac{\hbar^{2} k^{2}}{8 m^{2} c^{2}}-\frac{\hbar}{4 m c^{2}}(\boldsymbol{k}[\boldsymbol{\sigma}, \boldsymbol{u}])\right)+ \\
& +\frac{1}{c k}\left([\boldsymbol{k}, \boldsymbol{u}]+\frac{\hbar}{2 m}[\boldsymbol{k}[\boldsymbol{k}, \boldsymbol{\sigma}]]\right), \\
& \boldsymbol{r}(\tau)=\boldsymbol{r}-\hbar \int_{\tau}^{\beta} \mathrm{d} \tau^{\prime} \boldsymbol{u}\left(\tau^{\prime}\right), \quad \mathcal{D}^{3} u(\tau)=\prod_{0 \leqslant \tau \leqslant \beta} \mathrm{d}^{3} u(\tau) .
\end{aligned}
$$

The trace operation "Sp" in equation (3) is carried out over spin states, $\boldsymbol{A}_{\boldsymbol{k}}^{0}$ is the Fourier transform of $\boldsymbol{A}^{0}(\boldsymbol{r}), V$ is the system volume, $C$ is a normalizing factor. The functional (3) in the case, when the spin interactions are neglected and external field is absent, was found in paper [5].

Let us restrict ourselves by the random phase approximation (RPA) for the calculation of $\Omega$. For this purpose it is sufficient to take into account in $Z_{1}(\beta n)$ only the terms that are proportional to $e^{2}$. The calculation of path integrals is done according to

$$
\begin{aligned}
& \operatorname{reg} C \int \mathcal{D} u(\tau)\left(\cdots u\left(\tau_{0}\right) \cdots\right) \exp \int_{0}^{\beta} \mathrm{d} \tau\{\mathcal{L}(i u(\tau))-i P(\tau) u(\tau)\}= \\
& =\left.\operatorname{reg}\left(\cdots \frac{1}{i} \frac{\delta}{\delta \varphi\left(\tau_{0}\right)} \cdots\right) \exp \int_{0}^{\beta} \mathrm{d} \tau \mathcal{H}(P(\tau)-\varphi(\tau))\right|_{\varphi=0},
\end{aligned}
$$

where $\mathcal{H}(p)$ is the Hamiltonian function that corresponds to Lagrangian $\mathcal{L}(v)$. After calculations that are omitted here one obtains

$$
\begin{aligned}
& -\beta \Omega(R)=\sum_{\boldsymbol{p}, s} \ln \left(1+\mathrm{e}^{\beta\left(\mu-\varepsilon_{p}\right)}\right)+ \\
& +\frac{\beta}{2} \sum_{\boldsymbol{k}}\left(\frac{4 \pi e^{2}}{V k^{2}}\right) \sum_{\boldsymbol{p}, s}\left\langle n_{\boldsymbol{p}}\right\rangle\left(1-\frac{p^{2}-(\boldsymbol{k}, \boldsymbol{p})^{2} / k^{2}}{m^{2} c^{2}}-3 \Lambda_{k}\right)+ \\
& +\frac{1}{2} \sum_{\boldsymbol{k}}\left(\frac{4 \pi e^{2}}{V k^{2}}\right) \int_{0}^{\beta} \mathrm{d} \tau \int_{0}^{\beta} \mathrm{d} \tau^{\prime} \widetilde{R}_{\boldsymbol{k}}^{\mu}(\tau) \widetilde{R}_{-k}^{\mu}\left(\tau^{\prime}\right) \times \\
& \times\left\{P_{\boldsymbol{k}}^{\mu \nu}\left(\tau-\tau^{\prime}\right)+\frac{\delta\left(\tau-\tau^{\prime}\right)}{m c^{2}} l_{\mu \nu}^{\perp} \sum_{\boldsymbol{p}, s}\left\langle n_{\boldsymbol{p}}\right\rangle\right\}
\end{aligned}
$$


where

$$
\begin{aligned}
& P_{\boldsymbol{k}}^{\mu \nu}\left(\tau-\tau^{\prime}\right)=\sum_{\boldsymbol{p}, s} \mathrm{e}^{\left|\tau-\tau^{\prime}\right| \Delta \varepsilon}\left\langle n_{\boldsymbol{p}}\right\rangle\left(1-\left\langle n_{\boldsymbol{p}-\hbar \boldsymbol{k}}\right\rangle\right) \times \\
& \times\left\{l_{\mu \nu}^{\|}\left(1-\Lambda_{k}\right)-l_{\mu \nu}^{\perp}\left(\frac{p^{2}-(\boldsymbol{k}, \boldsymbol{p})^{2} / k^{2}}{m^{2} c^{2}}+\Lambda_{k}\right)\right\}, \\
& \Lambda_{k}=\frac{\hbar^{2} k^{2}}{4 m^{2} c^{2}}, \quad \widetilde{\boldsymbol{R}}_{\boldsymbol{k}}^{\mu}(\tau)=\boldsymbol{R}_{\boldsymbol{k}}^{\mu}(\tau)+\frac{1}{\sqrt{4 \pi V}}\left[\boldsymbol{k}, \boldsymbol{A}_{\boldsymbol{k}}^{0}\right], \\
& \Delta \varepsilon=\varepsilon_{p}-\varepsilon_{|\boldsymbol{p}-\hbar \boldsymbol{k}|}, \quad \varepsilon_{p}=\frac{p^{2}}{2 m}-\frac{p^{4}}{8 m^{3} c^{2}}, \\
& l_{\mu \nu}^{\|}=k_{\mu} k_{\nu} / k^{2}, \quad l_{\mu \nu}^{\perp}=\delta_{\mu \nu}-k_{\mu} k_{\nu} / k^{2},
\end{aligned}
$$

$\left\langle n_{\boldsymbol{p}}\right\rangle$ is the Fermi-Dirac distribution.

Thus, within RPA the path integral (2) is of Gaussian type. After this calculation one finds the thermodynamical potential $\Omega$. In particular, the corrections to $\Omega$ due to the interaction of particles with the magnetic fields are given by the formula

$$
\begin{aligned}
& \Delta \Omega^{m g}=\frac{1}{2} \sum_{\boldsymbol{k}}\left\{\left(\boldsymbol{A}_{\boldsymbol{k}}^{0}, \boldsymbol{A}_{-k}^{0}\right)-\left(\boldsymbol{k}, \boldsymbol{A}_{\boldsymbol{k}}^{0}\right)\left(\boldsymbol{k}, \boldsymbol{A}_{-k}^{0}\right) / k^{2}\right\} \times \\
& \times \frac{\frac{e^{2}}{V}\left[\frac{1}{m c^{2}} \sum_{\boldsymbol{p}, s}\left\langle n_{\boldsymbol{p}}\right\rangle+P_{\boldsymbol{k}, 0}^{\perp}\right]}{1+\frac{4 \pi e^{2}}{V k^{2}}\left[\frac{1}{m c^{2}} \sum_{\boldsymbol{p}, s}\left\langle n_{\boldsymbol{p}}\right\rangle+P_{\boldsymbol{k}, 0}^{\perp}\right]},
\end{aligned}
$$

where $P_{k, 0}^{\perp}=\left.P_{k, n}^{\perp}\right|_{n=0}, P_{k, n}^{\perp}$ are Fourier transforms of the transversal parts of the propagator $P_{\boldsymbol{k}}^{\mu \nu}\left(\tau-\tau^{\prime}\right): P_{\boldsymbol{k}, n}^{\perp}=\Pi_{\boldsymbol{k}, n}^{\perp}-\Lambda_{k} \Pi_{\boldsymbol{k}, n}^{\|}$. If $n=0$ then

$$
\begin{aligned}
& \Pi_{\boldsymbol{k}, 0}^{\|}=-2 \sum_{\boldsymbol{p}, s}\left\langle n_{\boldsymbol{p}}\right\rangle /|\Delta \varepsilon| \\
& \Pi_{\boldsymbol{k}, 0}^{\perp}=\frac{1}{(m c)^{2}} \sum_{\boldsymbol{p}, s}\left(p^{2}-\frac{(\boldsymbol{k}, \boldsymbol{p})^{2}}{k^{2}}\right)\left\langle n_{\boldsymbol{p}}\right\rangle /|\Delta \varepsilon| .
\end{aligned}
$$

Below, we shall describe the case of the constant homogeneous magnetic field $H_{0 x}=H_{0 y}=0, H_{0 z}=H_{0}$. Taking into account the Coulomb gauge $\left(\boldsymbol{k}, \boldsymbol{A}_{\boldsymbol{k}}^{0}\right)=0$ and equalities $\delta^{\prime}(z)=\delta(z) / z, \delta^{(3)}(0)=V /(2 \pi)^{3} \quad(V \rightarrow \infty)$ one can show that $\left(\boldsymbol{A}_{\boldsymbol{k}}^{0}, \boldsymbol{A}_{-k}^{0}\right)=(2 \pi)^{3} V H_{0}^{2} \delta(k) / k^{2}$. Then

$$
\Delta \Omega^{m g}=\frac{V H_{0}^{2}}{8 \pi} \lim _{\boldsymbol{k} \rightarrow 0}\left(\frac{4 \pi e^{2}}{V k^{2}}\right) \frac{\frac{1}{m c^{2}} \sum_{\boldsymbol{p}, s}\left\langle n_{\boldsymbol{p}}\right\rangle+\Pi_{\boldsymbol{k}, 0}^{\perp}-\Lambda_{k} \Pi_{\boldsymbol{k}, 0}^{\|}}{1+\frac{4 \pi e^{2}}{V k^{2}}\left[\frac{1}{m c^{2}} \sum_{\boldsymbol{p}, s}\left\langle n_{\boldsymbol{p}}\right\rangle+\Pi_{\boldsymbol{k}, 0}^{\perp}-\Lambda_{k} \Pi_{\boldsymbol{k}, 0}^{\|}\right]} .
$$

In the case of absolute zero one can get

$$
\Pi_{\boldsymbol{k}, 0}^{\|}=\frac{V k_{0} m}{\pi \hbar^{2}}, \quad \Pi_{\boldsymbol{k}, 0}^{\perp}=-\frac{V k_{0}^{3}}{3 \pi^{2} m c^{2}}\left(1-\frac{k^{2}}{4 k_{0}^{2}}\right),
$$


where $\hbar k_{0}$ is the marginal momenta, connected with chemical potential by the condition $\varepsilon_{\hbar k_{0}}=\mu$. Using these results in (6) and neglecting the spin interaction contribution $\left(\Lambda_{k}=0\right)$, we find

$$
\Delta \Omega^{d i a}=\frac{V H_{0}^{2}}{8 \pi} \frac{e^{2} k_{0} / 3 \pi m c^{2}}{1+e^{2} k_{0} / 3 \pi m c^{2}} .
$$

Expression (7) describes the contribution of diamagnetic effects into $\Omega$. Taking into account the spin interactions we obtain

$$
\Delta \Omega^{m g}=-\frac{m V}{12 \pi^{2}} k_{0}\left(\frac{e H_{0}}{m c}\right)^{2} /\left(1-\frac{2 e^{2} k_{0}}{3 \pi m c^{2}}\right) .
$$

The last expression accounts diamagnetism of Landau as well as paramagnetism of Pauli. From this expression we find the magnetization and magnetic susceptibility

$$
\boldsymbol{M}=\chi \boldsymbol{H}_{0}, \quad \chi=\frac{e^{2} k_{0}}{6 \pi^{2} m c^{2}} /\left(1-\frac{2 e^{2} k_{0}}{3 \pi m c^{2}}\right) .
$$

The dependence on the marginal momenta $\hbar k_{0}$ is excluded on the basis of the equation for chemical potential. We restrict ourselves to a nonrelativistic value of $\hbar k_{0}$. Then

$$
\begin{aligned}
\frac{\chi}{\chi_{0}} & =\frac{1}{1-a \alpha^{2} / r_{S}}+\frac{c_{0} r_{S}+c_{1} r_{S}^{2}+c_{2} r_{S}^{2} \ln r_{S}}{\left(1-a \alpha^{2} / r_{S}\right)^{2}}-c_{3} r_{S}^{4} \frac{a_{0}^{3} H_{0}^{2} / m c^{2}}{\left(1-a \alpha^{2} / r_{S}\right)^{4}} \\
\chi_{0} & =\frac{a}{4 \pi} \frac{\alpha^{2}}{r_{S}} .
\end{aligned}
$$

Here $\alpha=e^{2} / \hbar c$ is the fine structure constant, $a_{0}=\hbar / m e^{2}$ is Bohr radius, $a \approx 0,407 ; c_{0} \approx 0,166 ; c_{1} \approx-0,021 ; c_{2} \approx 0,008 ; c_{3} \approx 0,006$.

Equations (6) - (8), (10) are correct in the region of the weakly external field and high electron density that corresponds to the small values of Brueckner parameter $r_{S}\left(\alpha^{2} \ll r_{S} \ll 1\right)$. In the non-relativistic limit the equations (6) - (8), (10) are in agreement with the known results [3]. As one can see from (10) the exchange corrections, that are proportional to coefficient $c_{0}$ and relativistic contributions due to constant $\alpha$ (in contrast to correlation corrections connected with $c_{1}$ and $c_{2}$ ), lead to the increase of magnetic susceptibility. In the region of high electron density the relativistic corrections are essential. They may be to great extent larger than the nonrelativistic correlation contributions and approach to exchange corrections with respect to magnitude of order.

\section{References}

[1] Frankel N.E., Opat G.I., Spitzer J.J. Exact statistical mechanics of a relativistic magnetic anomaly. // Phys. Lett. A, 1967, vol. 25, No 10, p. 716-717.

[2] Delsante A.E., Frankel N.E. Relativistic paramagnetism: Quantum statistics. // Phys. Rev. D, 1979, vol. 20, No 8, p. 1795-1806.

[3] White R.M. Quantum theory of magnetism. New York, Mc. Graw-Hill Book Company, 1970. 
[4] Blazhyjevskii L.F. Path Integrals in configuration space in the weakly relativistic many bodies theory. // Teor. Mat. Fiz., 1986, vol. 66, No 3, p. 409-421, (in Russian).

[5] Blazhyjevskii L.F. The Quasirelativistic collective variables and path integrals in statistical mechanics of degenerated systems. // Ukr. Fiz. Zhurn., 1986, vol. 31, No 2, p. 297-303, (in Russian).

\title{
БАГАТОЧАСТИНКОВІ КОРЕЛЯЦІІ ТА МАГНЕТИЗМ СЛАБОРЕЛЯТИВІСТСЬКОГО ЕЛЕКТРОННОГО ГАЗУ
}

\author{
Л.Ф.Блажиєвський, Г.Б.Гіль, Ю.С.Криницький
}

На основі методу континуального інтегрування досліджуються магнітні властивості слаборелятивістського електронного газу у неоднорідному магнітному полі. Обчислено термодинамічний потенціал у наближенні хаотичних фаз, знайдено намагніченість і нелінійну магнітну сприйнятливість при температурі абсолютного нуля, де було враховано діамагнетизм Ландау та парамагнетизм Паулі. Показано, що у випадку високої густини електронного газу релятивістські внески є суттєвими і ведуть до зростання магнітної сприйнятливості. 